\title{
Recurrent haematomas of the thigh: a case of von Willebrand's disease presenting to a sports clinic
}

\author{
Simon Owens, Trevor Baglin
}

\begin{abstract}
Von Willebrand's disease is a relatively common mild form of haemophilia. It should be suspected in assessing sports injuries when excessive bleeding occurs in response to relatively mild trauma. Those with the disease should remain active but avoid contact sports. They should not take aspirin or non-steroidal antiinflammatory drugs, which may exacerbate bleeding, and should be given supportive treatment to cover dental extraction, surgery, or significant bleeding episodes.
\end{abstract}

(Br F Sports Med 2000;34:122-124)

Keywords: von Willebrand's disease; haemophilia; haematoma; sports clinic

\section{Case history}

A 19 year old college rugby player and rower presented to the sports injury clinic with a history of recurrent injuries to his left thigh. The initial injury had occurred at the age of 13 when he sustained a knock and subsequent haematoma while playing rugby. Physiotherapy was given and the injury took several weeks to settle. Again, one year before presentation he had sustained a similar recurrent blunt injury to the thigh followed by significant thigh swelling, which had taken four weeks to settle with physiotherapy. An $x$ ray showed no bony abnormality or myositis ossificans.

At presentation he had knocked the outer aspect of his left thigh on his desk five days earlier, which had left him with some mild muscle tenderness and discomfort but without any swelling. Three days later he had gone for a run, though he was still aware of some discomfort in the thigh. The following day he found increased pain and stiffness and movement was limited. He had not taken any antiinflammatory drugs or aspirin that might have exacerbated a bleeding tendency.

On examination he had a swollen thigh with

Peter Wilson Sports Clinic, Addenbrooke's NHS Trust, Hills Road, Cambridge CB2 2QQ, UK

S Owens

Clinical Haematology, Box 234, Addenbrooke's NHS Trust

T Baglin

Correspondence to: Dr Owens

Accepted for publication 5 October 1999 a $15 \times 20 \mathrm{~cm}$ swelling over the vastus lateralis, which was generally tender. There was a small sympathetic effusion in the left knee. He had limitation of knee flexion to $80^{\circ}$.

A further $x$ ray examination was made which showed some cortical thickening of the posterolateral aspect of the mid shaft of the femur consistent with old calcification of a subperiosteal haematoma. It was not thought likely that he had a stress fracture given the history of only mild trauma, and there was no evidence of myositis ossificans. A soft tissue ultrasound examination showed an $8 \times 3 \times 3 \mathrm{~cm}$ that the thigh had swelled up considerably with

haematoma and confirmed a bump in the contour of the femur in transverse section.

On further inquiry it was evident that the patient had tended to bruise easily in the past and had bled excessively on one occasion after a dental extraction, but had never undergone any other surgery or sought medical advice before this about a possible bleeding tendency. In view of this history of a possible bleeding diathesis a full blood count and clotting studies were undertaken which showed haemoglobin $144 \mathrm{~g} / 1$, white cell count $13.3 \times 10^{9} / 1$, platelets $360 \times 10^{9} / 1$, partial thromboplastin time 13.6 seconds (normal 12-16), activated partial thromboplastin time (APTT) 53.3 seconds (normal 28-46). Repeat clotting studies confirmed a prolonged APTT of 48.0 seconds. He was therefore referred to the haematology department, where a prolonged bleeding time of $>11$ minutes was confirmed (normal $<9$ minutes). Further clotting studies showed a von Willebrand's antigen of $27 \%$ (normal $>55 \%$ ) and ristocetin cofactor (RICOF) of $30 \%$ (normal $>55 \%$ ), confirming a diagnosis of mild von Willebrand's disease.

He was advised by the haematology department that in future he should avoid nonsteroidal anti-inflammatory drugs and aspirin, and that he would require treatment with desmopressin acetate (DDAVP) and tranexamic acid to cover dental extraction or any other surgery. Supportive treatment might also be given to cover any further significant bleeding episode. It was not thought that he would run into any problems in his day to day life. However, it was recommended that he should no longer participate in contact sport and he is now concentrating on his light-weight rowing. ${ }^{12}$

\section{Discussion}

Von Willebrand's disease is the most commonly inherited bleeding disorder and is the result of a deficiency or abnormality of an adhesive glycoprotein called von Willebrand's factor (VWF).

This factor is synthesised by megakaryocytes and is stored in platelet $\alpha$ granules. It is also produced by and stored in endothelial cells and present as large glycoprotein multimers in the circulation.

Deficiency of VWF causes a reduction in platelet adherence to damaged subendothelium and also in platelet-platelet aggregation, with a consequent increase in bleeding time (fig 1). In addition, the level of factor VIII, which is carried and stabilised by VWF, is often reduced.

The gene for VWF is on chromosome 12 and the disease is generally inherited as an 


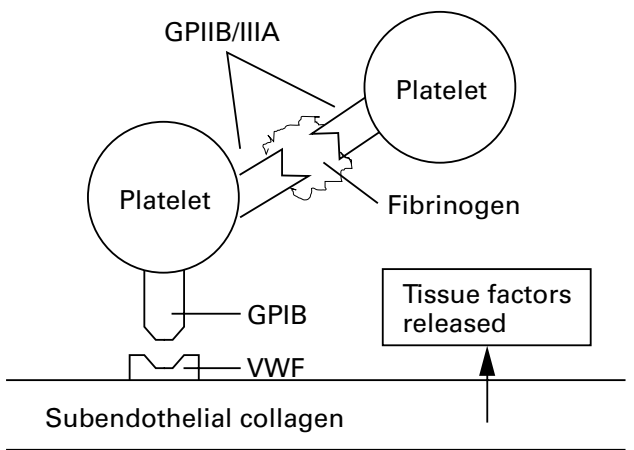

Figure 1 Role of von Willebrand's factor (VWF). 1. Tissue damage exposes subendothelial collagen together with $V W F$ bound to it. It also releases tissue factors that activate intrinsic and extrinsic coagulation pathways. 2. VWF binds to platelet glycoprotein IB (GPIB), thus allowing the platelet to adhere to the damaged subendothelium. 3. This causes a change in platelet shape and release from within the platelet of further VWF from a storage granules. 4. Platelet adhesion causes a conformational change in the fibrinogen receptor (GPIIB/IIIA) which increases its affinity, resulting in platelet aggregation through fibrinogen cross linking of platelets.

autosomal dominant condition with an overall prevalence in the population of at least $0.8 \%$. However, there is a wide phenotypic heterogeneity. Patients usually have a mild to moderate bleeding diathesis, mainly manifest in mucocutaneous bleeding or post-traumatic bleeding. Commonly, it presents after dental extraction, epistaxis, menorrhagia, postpartum haemorrhage, or with easy bruising. This form of haemophilia tends to be mild compared with haemophilia A (due to the deficiency in factor VIII) and haemophilia B (Christmas disease due to factor IX deficiency), which tend to present with major bleeding into muscle and joints that can lead to joint damage, synovitis, and contracture. ${ }^{1-3}$

Diagnosis of von Willebrand's disease, especially in its milder forms, may be difficult and there are several subtypes distinguished by haematological quantitative and qualitative assays. In mild von Willebrand's disease both the APTT and the bleeding time may be normal. Therefore a strong personal history of bleeding is an indication for direct measurement of von Willebrand protein levels, by both antigenic (ELISA) and functional RICOF assays. It is essential to take blood with the patient in a resting state, ideally without a tourniquet. It may be necessary to take serial blood samples to document a low level. Given the large size of the VWF gene and the large number of mutations that have been described, routine genetic analysis is not currently feasible.
This case presented with recurrent haematomas after relatively mild trauma. The possibility of a bleeding diathesis should always be considered in such cases, and clues to this should be sought in the history. Von Willebrand's disease may present, as in this case, with relatively mild symptoms, but other conditions such as acute compartment syndrome after venepuncture and epidural haematoma after minor trauma have been associated with the disease. ${ }^{4-6}$ It may also present relatively late in adolescence or early adulthood compared with the major clotting disorders that tend to present in early childhood. The benefits of picking up a coagulation defect are obviously important in preventing serious haemorrhage due to trauma and complications related to surgery in the future.

A review of sports and haemophilia in 1996 concluded that those with major haemophilia tended to be overprotected and were generally not allowed to participate in sporting activities normal to their peer group. ${ }^{7}$ It was recommended that subjects with haemophilia $\mathrm{A}$ and B should generally be encouraged to be active as there was some evidence that exercise might reduce or prevent intra-articular haemorrhage by maintaining muscle strength and tone. Contact sports were generally discouraged, but other sports such as swimming, golf, and table tennis were encouraged. The advice in von Willebrand's disease is less clear cut as there is considerable phenotypic variability in the severity of the disease. It would be prudent to obtain individual advice from the local haematology department, and avoid contact sports if there is any significant bleeding diathesis.

Dr Simon Owens initially managed and investigated this case at presentation and then referred him on to Dr Trevor Baglin for specialist haematological assessment. Dr Simon Owens and Dr Baglin collaborated in producing the paper and both accept responsibility as guarantors for this case report. We would like to thank Michelle Cornell for preparing fig 1 .

1 Castaman G, Rodeghiero F. Current management of von Willebrand's disease. Practical therapeutics. Drugs 1995;50:602-14

2 Von Willebrand working party. Guidelines for the diagnosis and management of Von Willebrand's disease. UK: Haemophilia Centre Directors Organisation, 1995.

3 Venkateswaran L, Wilimas JA, Jones DJ, et al. Mild hemophilia in children: prevalence, complications and reatment. F Pediatr Hematol Oncol 1998;20:32-5.

4 Takenaka N, Mine T, Ikeda E, et al. Acute epidural haematoma of the posterior fossa in a case of von Willebrand's disease. No-Shinkei-Geka 1988;16(suppl 5): 529-33.

5 Nixon RG, Brindley GW. Haemophilia presenting as compartment syndrome in the arm following venepuncture. A case report and review of the literature. Clin Orthop

6 Tountas CP, Ferris FO, Cobb SW. Exertional compartment syndrome in covert mild haemophilia. A case report. MinnMed 1992;75:27-9.

7 Buzzard B. Sports and haemophilia. Clin Orthop 1996;328: 25-30.

Take home message

Mild coagulation defects are relatively common and may present in late childhood or early adult life as a result of sporting injury. A bleeding diathesis should be suspected when disproportionate bruising and swelling occur in response to relatively minor trauma. 


\section{Commentary}

Bleeding problems presenting as a result of sporting injury are rare. They are either congenital or acquired and most congenital problems present in early childhood when toddlers begin to walk and fall over. There are, however, some disorders that are milder and which may only cause haematoma as a result of more severe injury; von Willebrand's disease is the commonest of these. There are also a number of hereditary platelet problems that may present with easy bruising and may require specialist referral for diagnosis. Most cases of easy bruising are not associated with any haematological abnormality. A bleeding time is a useful means of assessing such patients, but a detailed history, including family history, and aclinical examination, are prerequisite and are best undertaken by a specialist. Asprin and other non-steroidal anti-inflammatory drugs can lead to easy bruising in normal people. Thrombocytopenia is a fairly common condition, usually transient, which can cause easy bruising and infectious mononucleosis, and can occasionally result in severe thrombocytopenia, and splenic rupture as a result of playing rugby while convalescing from infectious mononucleosis has occurred.

Other coagulation problems fortunately are rare and usually associated with such severe illnesses that the patient would be too unwell to participate in sport.

\section{Exercise on both sides of the curriculum}

Dundee Medical School changed its curriculum five years ago to accommodate the General Medical Council publication Tomorrow's Doctors. We are in the early stages of creating a wellbeing programme for our medical students, which has only reached the pilot stage. This programme is based around an awareness raising instrument developed by the university counselling service which encourages medical students to examine their own wellbeing in the domains of mind, body, and spirit. These domains include the amount of exercise students take, how well they sleep, what type of relationships theyhave, how balancedtheir diet is, and how much they use stimulants such as caffeine. Students plot their own results and receive aggregated data from their class. Early aggregated results tend to show students scoring high in the areas of mind and spirit, eating a reasonably balanced diet, but exercising less than is ideal. Our instrument is still in the developmental stage and requires considerable work in terms of developing its validity and reliability parameters.

University of Dundee Counselling Services

NICK HALPIN

Tayside Centre for General Practice, DAVID SNADDEN University of Dundee 